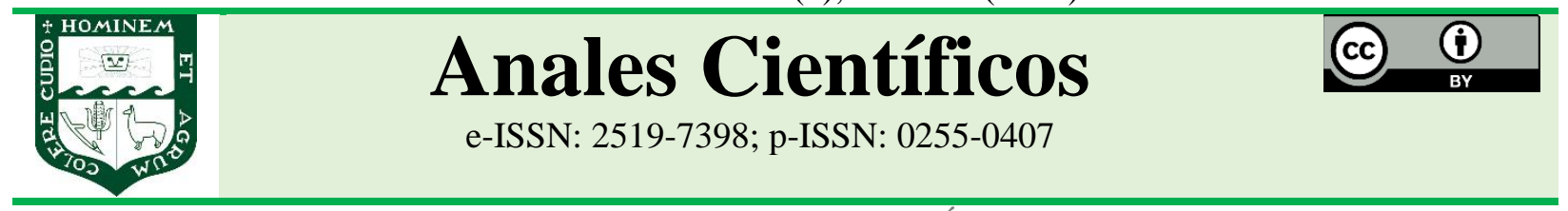

ARTÍCULO ORIGINAL - RESEARCH ARTICLE

http://dx.doi.org/10.21704/ac.v82i2.1792

\title{
ACTIVIDAD ENZIMÁTICA DE PALTA FUERTE (persea americana) DURANTE EL ALMACENAMIENTO EN REFRIGERACIÓN
}

\section{Enzymatic activity of avocado (persea americana) var. Fuerte during refrigerated storage}

\author{
Jhoselyn Liñan-Pérez ${ }^{1 *(D)}$; Gustavo Puma-Isuiza1 (iD) \\ ${ }^{1}$ Facultad de Industrias Alimentarias, Universidad Nacional Agraria La Molina, La Molina, 15024, Lima, Perú \\ * E-mail: gpuma@lamolina.edu.pe
}

Recibido: 14/09/2020; Aceptado: 05/10/2021; Publicado: 31/12/2021

\begin{abstract}
The aim of this study was to evaluate the effect of physical treatments (heat treatment at $45^{\circ} \mathrm{C}$ : TT), chemical (citric acid 1.0\%: AC 1.0\%; ascorbic acid $0.75 \%$ : AA $0,75 \%$ and $1.0 \%$ sodium tripolyphosphate: $1.0 \%$ TPS), combined $\left(0.2 \%\right.$ citric acid and $\left.45^{\circ} \mathrm{C}: 0.2 \% \mathrm{AC}+\mathrm{TT}\right)$ and a control treatment $(\mathrm{C})$, in the inhibition of enzymatic browning, measured through the enzymatic activity ( $\triangle$ absorbance/min) of Polyphenol Oxidase (PPO), in avocado tissue stored at refrigeration temperature $\left(4^{\circ} \mathrm{C}\right)$. The CIELAB color parameters $\left(\mathrm{L}^{*} \mathrm{a}^{*} \mathrm{~b}^{*}\right), \Delta \mathrm{E}^{*}, \Delta \mathrm{C}^{*}$ and the percentage of obscuration were evaluated. In addition, a sensory test was carried out with 50 consumers who evaluated the preference of the treated avocado samples. In general, the anti-browning treatments reduced the enzymatic activity of PPO where the sample treated with citric acid at $0.2 \%$ and $45{ }^{\circ} \mathrm{C}$ obtained the best results in reducing the enzymatic activity and the color parameters.
\end{abstract}

Keywords. Avocado | enzymatic browning | polyphenol oxidase | fourth range products | enzymatic activity

\section{RESUMEN}

El objetivo del presente estudio fue evaluar el efecto de tratamientos físicos (tratamiento térmico a $45{ }^{\circ} \mathrm{C}$ : TT), químicos (ácido cítrico al 1,0\%: AC 1,0 \%; ácido ascórbico al 0,75 \%: AA 0,75\% y Tripolifosfato de sodio al 1,0 $\%$ : TPS $1,0 \%$ ), combinados (ácido cítrico al $0,2 \%$ y $45^{\circ} \mathrm{C}$ : AC $0,2 \%+\mathrm{TT}$ ) y un tratamiento control (C), en la inhibición del pardeamiento enzimático, medido a través de la actividad enzimática $(\Delta$ absorbancia/min) de la Polifenol Oxidasa (PPO), en el tejido de palta almacenada a temperatura de refrigeración $\left(4^{\circ} \mathrm{C}\right)$. Se evaluaron los parámetros de color CIELAB $\left(\mathrm{L}^{*} \mathrm{a}^{*} \mathrm{~b}^{*}\right), \Delta \mathrm{E}^{*}, \Delta \mathrm{C}^{*}$ y el porcentaje de oscurecimiento. Además, se realizó una prueba sensorial con 50 consumidores quienes evaluaron la preferencia de las muestras de palta tratadas. En general, los tratamientos anti-pardeantes redujeron la actividad enzimática de la PPO donde la muestra sometida con ácido cítrico al $0,2 \%$ y $45{ }^{\circ} \mathrm{C}$ obtuvo los mejores resultados en la reducción de la actividad enzimática y los parámetros de color.

Palabras clave: aguacate | pardeamiento enzimático | polifenol oxidasa | productos de cuarta gama | actividad enzimática

Forma de citar el artículo (Formato APA):

Liñan-Pérez, J., \& Puma-Isuiza, G. (2021). Actividad enzimática de palta fuerte (persea americana) durante el almacenamiento en refrigeración. Anales Científicos. 81(2), 296-302. http://dx.doi.org/10.21704/ac.v82i2.1792

Autor de correspondencia (*): Gustavo Puma-Isuiza. Email: gpuma@lamolina.edu.pe

(C) Los autores. Publicado por la Universidad Nacional Agraria La Molina.

This is an open access article under the CC BY 


\section{INTRODUCCIÓN}

Los productos frescos trozados listos para consumir, se están volviendo muy populares entre los consumidores debido a su practicidad; sin embargo, el productor se enfrenta ante el desafío de mantener la calidad a lo largo de la vida útil del producto final (GonzálesAguilar et al., 2005) puesto que el procesamiento de frutas y verduras frescas cortadas promueve su deterioro fisiológico, bioquímico, sensorial y microbiano, incluso cuando se realizan procesos mínimos (Bustos et al., 2015).

El principal problema que afecta directamente a las propiedades del producto es el pardeamiento enzimático que se debe principalmente a la actividad catalítica de la Polifenol Oxidasa (PPO), que desempeña el papel central en el pardeamiento de alimentos recién cortados. Se trata de una metaloenzima oligomérica con un sitio activo que contiene dos iones de cobre, cada uno coordinado con tres residuos de histidina (Chang, 2009). La PPO, en presencia de oxígeno molecular, cataliza la reacción de oxidación de los o-difenoles, presentes en el tejido de las frutas y verduras, en o-quinonas. Luego estas oquinonas se polimerizan reaccionando con fenoles y otras sustancias para producir pigmentos de color marrón oscuro (Gacche et al., 2006; Yoruk \& Marshall, 2003) que afectan directamente al color del producto que es el primer atributo sensorial percibido por el consumidor (Hernández \& Briceño, 2009).

La palta (Persea americana Mill.) es una fruta climatérica altamente susceptible al pardeamiento enzimático y ha sido objeto de varios estudios donde se evaluaron diferentes compuestos antioxidantes sintéticos, temperaturas de almacenamiento y la aplicación de atmósferas modificadas para evitar el pardeamiento (Oms-Oliu et al., 2010; Soliva, Elez, Sebastián, \& Martín, 2001); sin embargo, la PPO de la palta tiene una alta actividad catalítica y una resistencia considerable a los tratamientos de inhibición comunes en comparación con las PPO de otras frutas (Toledo \& Aguirre, 2016; Weemaes et al., 1999), por esta razón su inhibición es un tema de investigación debido a la dificultad de preservar la calidad fresca de la palta durante períodos prolongados (Gendel, 2012).

Los compuestos fenólicos también son relevantes en términos del flavor de los alimentos, ya que pueden desempeñar un papel en el sabor amargo, dulce, picante $o$ astringente de algunos productos y también pueden contribuir al aroma. En la palta las enzimas fenólicas oxidativas tienen efecto importante en la degradación del ácido cafeico, por lo que es de suma importancia conocer el efecto en las propiedades sensoriales (Tomás-Barberán \& Espín, 2001).

El objetivo de la presente investigación fue evaluar el efecto de diferentes tratamientos para inhibir o retrasar el pardeamiento y la actividad de la PPO de la palta, variedad fuerte, cortada y almacenada en refrigeración $\left(4{ }^{\circ} \mathrm{C}\right)$.

\section{MATERIALES Y MÉTODOS}

\section{Tratamiento de las muestras}

Los análisis se realizaron en los laboratorios de Biotecnología, Investigación e instrumentación y Evaluación sensorial de alimentos, pertenecientes a la Facultad de Industrias Alimentarias de la Universidad Nacional Agraria La Molina. Las paltas variedad Fuerte fueron lavadas por inmersión durante dos minutos en una solución de hipoclorito de sodio (200 $\mathrm{ppm})$, luego se pelaron, deshuesaron y cortaron en rodajas (Denoya et al., 2012; Soliva et al., 2001). Después, fueron sometidas durante cinco minutos a un baño de inmersión con diferentes agentes inhibidores de la actividad de la PPO (Tabla 1). Fueron envasadas en material de polietileno de alta densidad $(10 \times 10 \mathrm{~cm})$ y almacenadas en refrigeración $\left(4^{\circ} \mathrm{C}\right)$.

Tabla 1. Tratamiento de las muestras de palta refrigeradas a $4^{\circ} \mathrm{C}$

\begin{tabular}{ll}
\hline \multicolumn{1}{c}{ Tratamiento } & \multicolumn{1}{c}{ Descripción } \\
\hline $\mathrm{TT}^{*}$ & Agua destilada a $45^{\circ} \mathrm{C}$ \\
$\mathrm{AC} 0,2 \%+\mathrm{TT}^{*}$ & Solución de ácido cítrico al $0,2 \%$ a $45{ }^{\circ} \mathrm{C}$ \\
$\mathrm{AC}$ al $1,0 \%$ & Solución de ácido cítrico al $1,0 \%$ \\
$\mathrm{AA} 0,75 \%$ & Solución de ácido ascórbico al $0,75 \%$ \\
$\mathrm{TPS} 1,0 \%$ & Solución de tripolifosfato de sodio al 1,0 \\
& $\%$ \\
$\mathrm{C}$ & Agua destilada (muestra control) \\
\hline
\end{tabular}

$\mathrm{TT}^{*}$ : tratamiento térmico

\section{Medición del color}

Se delimitó un área de $5 \times 5 \mathrm{~cm}$ del envase que contenía la palta en rodajas, en donde se midieron las características cromáticas del espacio $L^{*}, a^{*}$ y $b^{*}$ 
(Minolta, CR 400) durante el almacenamiento en refrigeración por 15 días, mediante tres mediciones sobre el tejido amarillo (Woolf et al., 2013). La diferencia del color total $\left(\Delta \mathrm{E}^{*}\right)$, el cambio de los aspectos cromáticos $\left(\Delta \mathrm{C}_{\mathrm{ab}}\right)$ y el porcentaje de oscurecimiento en función de $\mathrm{L}^{*}$ se determinó mediante las ecuaciones (1), (2) y (3), respectivamente (Bustos et al., 2015; Lu, Luo, Turner, \& Feng, 2007; Soliva et al. 2001).

$$
\begin{aligned}
& \Delta \mathrm{E}^{*}=\left[\left(\Delta \mathrm{L}^{*}\right)^{2}+\left(\Delta \mathrm{a}^{*}\right)^{2}+\left(\Delta \mathrm{b}^{*}\right)^{2}\right]^{0.5} \\
& \Delta \mathrm{C}_{a b}^{*}=\left[\left(\Delta \mathrm{a}^{*}\right)^{2}+\left(\Delta \mathrm{b}^{*}\right)^{2}\right]^{0.5} \\
& \% \text { pardeamiento }==\frac{\left|L_{\text {día } 0}^{*}-L_{\text {día } n}^{*}\right|}{L_{\text {control día } n}^{*}} \times 100
\end{aligned}
$$

Medición de la actividad enzimática de la polifenoloxidasa

Se mezclaron $25 \mathrm{~g}$ de puré de palta con $25 \mathrm{ml}$ de solución buffer McIlvaine ( $\mathrm{pH}$ 6,5). La mezcla homogeneizada fue centrifugada a $12000 \mathrm{rpm}$ durante 30 minutos a $4{ }^{\circ} \mathrm{C}$. El residuo sólido fue desechado y el sobrenadante fue filtrado a través de papel Whatman $\mathrm{N}^{\mathrm{o}}$ 4. El líquido resultante constituyó el extracto enzimático (EE) (Hernández \& Briceño, 2009; Soliva et al., 2001).

La actividad enzimática se determinó a través de la densidad óptica (DO). Se colocó $3 \mathrm{ml}$ de solución de catecol $0.02 \mathrm{M}$ (sustrato) en una cubeta de vidrio de 1 cm de ancho y se agregaron $70 \mu$ de extracto enzimático (EE). Se realizaron lecturas de la absorbancia a $410 \mathrm{~nm}$ cada 10 segundos durante 3 minutos. La actividad enzimática $(\Delta \mathrm{DO} / \mathrm{min})$ fue calculada mediante una regresión lineal de la parte inicial de la curva absorbancia vs tiempo de reacción (Hernández \& Briceño, 2009).

\section{Evaluación sensorial}

Se efectuó la prueba de preferencia ampliada (ranking) con 50 consumidores quienes ordenaron las muestras según su preferencia. Se asignó al primer, segundo, tercer y cuarto orden de preferencia el puntaje de 1, 2, 3 y 4, respectivamente (Moskowits et al., 2012).

\section{Análisis estadístico}

Se realizó un análisis de varianza $(\mathrm{p}<0,05)$ mediante un DBCA con tres repeticiones. Los datos generados en la evaluación sensorial se evaluaron con el estadístico de Friedman y la prueba de comparación de Wilcoxon. Todos los análisis estadísticos se llevaron a cabo con el software R versión 3.5.3.

\section{RESULTADOS Y DISCUSIÓN}

\section{Evaluación del color \\ Parámetros de color CIE L*, a* y b*}

La Figura 1 (A-C) muestra la luminosidad ( $\left.\mathrm{L}^{*}\right)$, el enrojecimiento $\left(a^{*}\right)$ y el color amarillento $\left(b^{*}\right)$ de las muestras de palta en diferentes tiempos de almacenamiento refrigerado $\left(4^{\circ} \mathrm{C}\right)$.

En la muestra no tratada (C), el almacenamiento en refrigeración provocó una reducción significativa $(\mathrm{p}<$ $0,05)$ de la luminosidad $\left(\mathrm{L}^{*}\right)$ y el color amarillento $\left(\mathrm{b}^{*}\right)$ durante las dos semanas (figura 1A y 1C) evidenciando la acción de la Polifenol Oxidasa (PPO) en la pérdida de luminosidad y del verdor que está asociado a la degradación de clorofilas que se produce durante el pardeamiento de la palta (Watada et al., 1990).

La muestra con adición de ácido cítrico al 0,2 \% y escaldada a $45^{\circ} \mathrm{C}(\mathrm{AC} 0,2 \%+\mathrm{TT})$ mostró una menor disminución significativa $(\mathrm{p}<0,05)$ de $\mathrm{L}^{*} \mathrm{y} \mathrm{b}^{*}$ (Figura 1 A-B) que la muestra tratada solamente con ácido cítrico al 1,0\% (AC al 1,0\%) evidenciando que existió un efecto combinado en la reducción del pardeamiento enzimático; sin embargo, se observó entre ambas muestras una tendencia similar respecto a la tasa de cambios de a* durante los 15 días (Figura 1C).

Los seis tratamientos tienen tendencia creciente en cuando al valor del cromático a* que está asociado sensorialmente al color verde (-a) y rojo (+a). Las muestras $\mathrm{C}$ y $\mathrm{TT}$ disminuyen rápidamente el verdor $\left(a^{*}\right)$. El valor de $a^{*}$ tiene a aumentar durante el almacenamiento; es decir disminuye la intensidad del color verde, siendo más pronunciado este efecto en los tratamientos que no llevan aditivos; esto debido a la producción de melaninas o melanoidinas, responsables de la coloración oscura marrón-rojiza característica del pardeamiento enzimático (Gacche et al., 2006). 

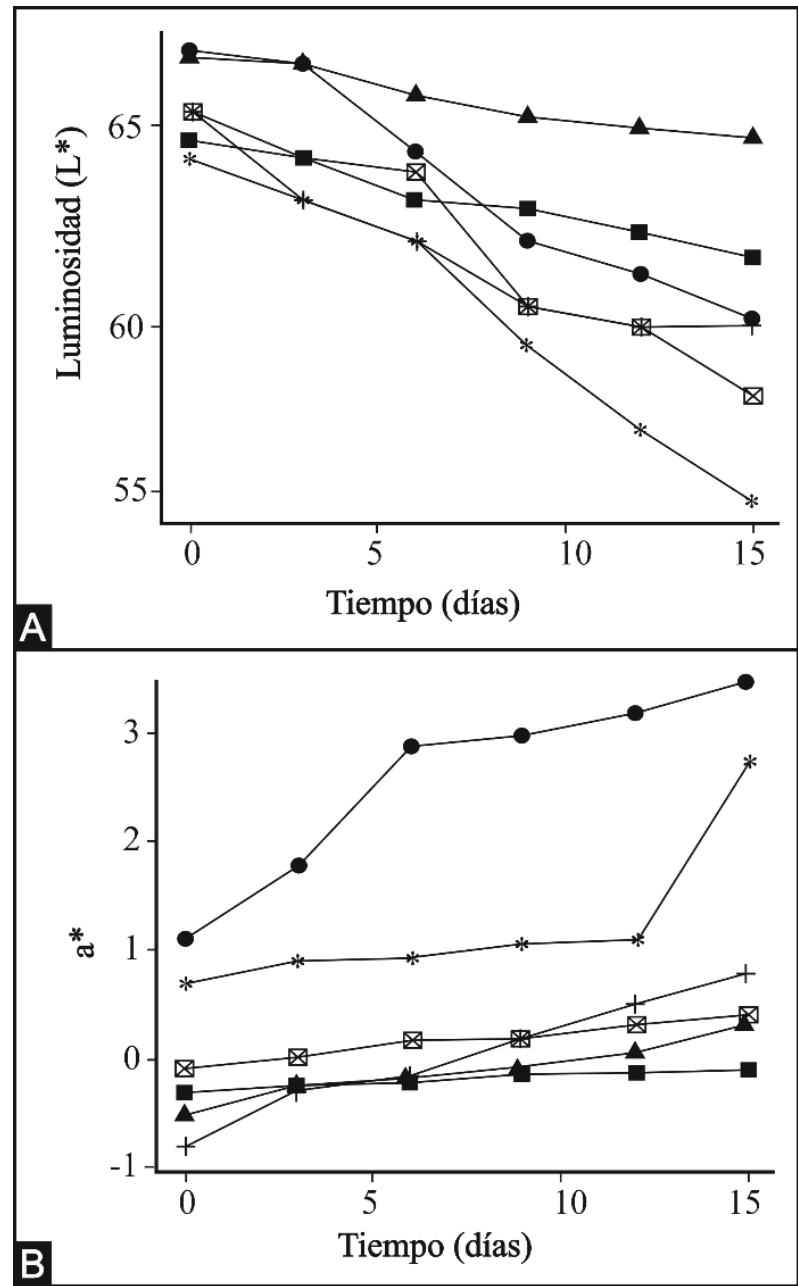

B

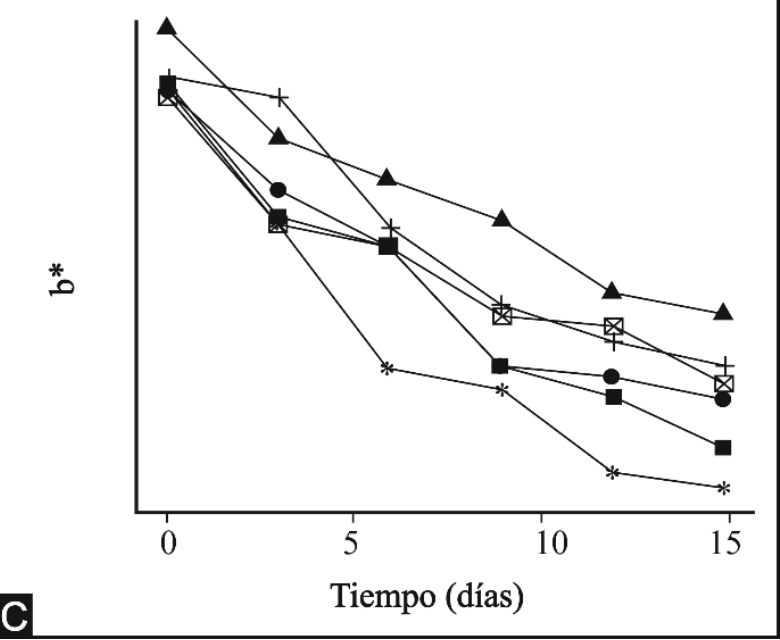

Figura 1. Luminosidad-L* (A), disminución del verdor-a* (B) y amarillez-b* (C) de muestras de palta refrigeradas a $4{ }^{\circ} \mathrm{C}$. Los símbolos en la Figura $1(\mathrm{~A}-\mathrm{C})$ indican, $\bullet:$ TT, $\mathbf{\Delta}:$ AC $0,2 \%+$ TT, $\mathbf{~ : ~ A C ~ a l ~ 1 , 0 \% , + : ~}$ AA $0,75 \%, \otimes:$ TPS $1,0 \%$ y $*$ C.
Variación del color total $\left(\Delta \mathbf{E}^{*}\right)$, aspectos cromáticos $\left(\Delta C_{a b} *\right)$ y porcentaje de oscurecimiento

La Figura 2 (A-C) muestra los resultados del cambio de color total, cambio de los aspectos cromáticos y del porcentaje de oscurecimiento de los seis tratamientos, medidos los días de almacenamiento 3, 9 y 15 respecto al día 0 .

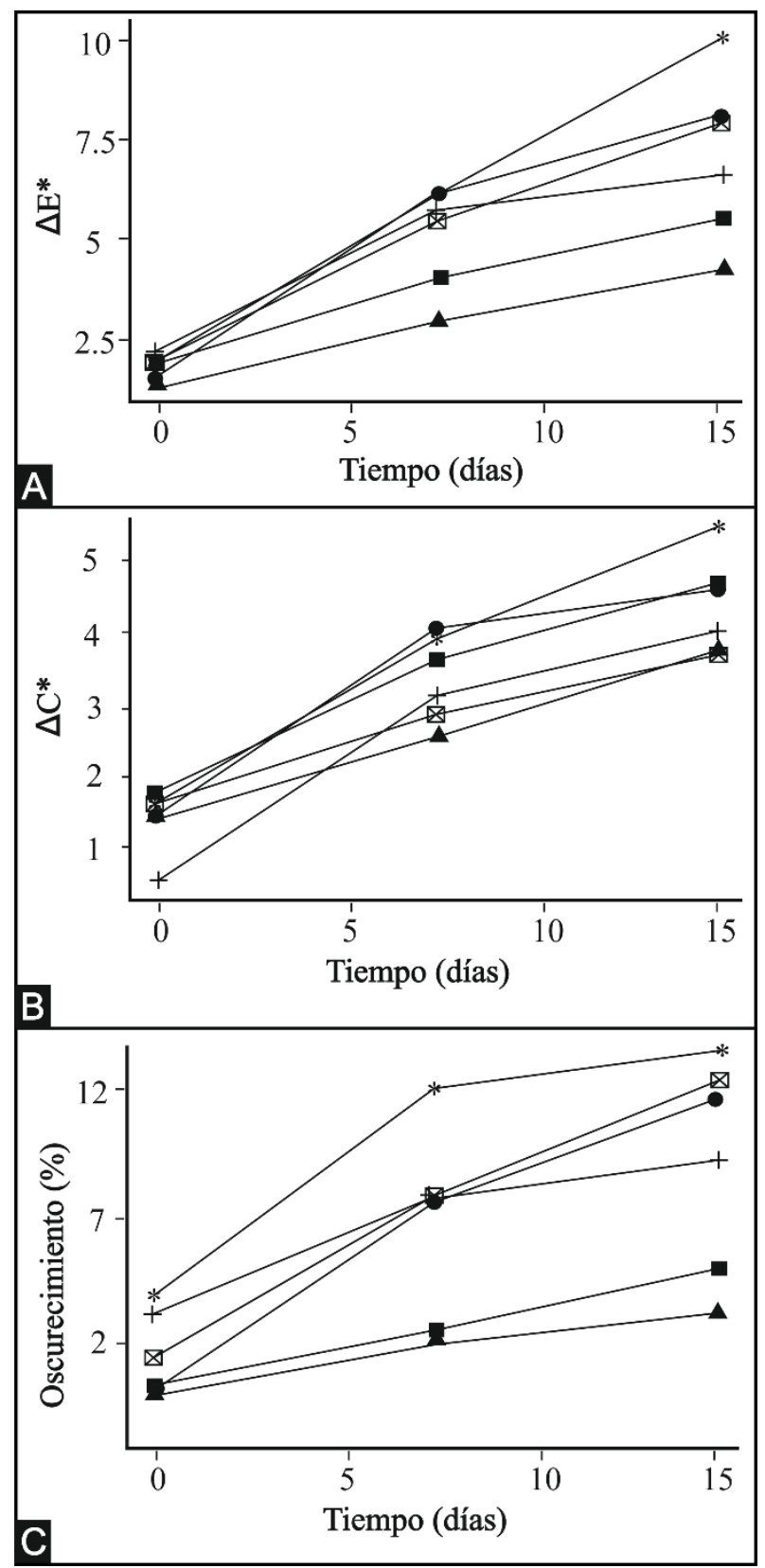

Figura 2. Cambio de color- $\Delta \mathrm{E}^{*}$ (A), aspectos cromáticos- $\Delta \mathrm{C}^{*} \quad$ (B) y oscurecimiento-\% $\quad$ (C) de muestras de palta refrigeradas a $4{ }^{\circ} \mathrm{C}$. Los símbolos en la Figura 2 (A-C) indican, •: TT, $\mathbf{\Delta}$ : AC 0,2 \% + TT, a: AC $1,0 \%,+$ : AA $0,75 \%, \bigotimes$ : TPS $1,0 \%$ y *: C. 
En todos los casos se observa que la muestra $\mathrm{C}$ obtuvo los valores más altos de $\Delta \mathrm{E}^{*}, \Delta \mathrm{C}^{*}$ y el porcentaje de oscurecimiento a comparación de las muestras sometidas a los tratamientos físicos, químicos o combinados, evidenciando un efecto en el pardeamiento enzimático. La muestra AC $0,2 \%+\mathrm{TT}$ tuvo el menor cambio total del color $\left(\Delta \mathrm{E}^{*}\right)$ seguido del tratamiento AC 1,0\% tanto en el día 9 como en el día 15. Con relación al cambio de los aspectos cromáticos (Figura $1 \mathrm{~B}$ ), de menor a mayor se obtuvo a las muestras AC 0,2 \% + TT, TPS $1,0 \%$, AA 0,75\%, TT, AC $1,0 \%$, y C. Además, el menor porcentaje de oscurecimiento de la muestra AC 0,2\% + TT (Figura 2C) está relacionado con los resultados obtenidos de la luminosidad-L* (Figura $1 \mathrm{~A}$ ).

Actividad enzimática de la Polifenol Oxidasa (PPO) La actividad enzimática de la PPO (Figura 3), de las seis muestras de palta, expresada en $\Delta$ absorbancia/min aumenta en función del tiempo de almacenamiento en refrigeración a $4{ }^{\circ} \mathrm{C}$. Esto puede deberse a que durante el almacenamiento la PPO sigue transformándose de su estado latente a activo incrementado su concentración y produciendo el pardeamiento enzimático (Palou et al., 1999).

La muestra Control (C), fue la que obtuvo mayor actividad enzimática a diferencia de las muestras que fueron sometidas a diferentes tratamientos de antipardeamiento enzimático con aditivos o mediante tratamiento térmico. La muestra AC $0,2 \%+$ TT tuvo menor actividad enzimática en el día 0 , seguida de la muestra AA $0,75 \%$, AC 1,0 \%, TPS $1,0 \%$. Esta tendencia es similar durante el tiempo de almacenamiento, a diferencia al día 15 en donde la curva de la muestra AA $0,75 \%$ tiene a ser horizontal al igual que las curvas de las muestras AC $0,2 \%+$ TT y AC $1,0 \%$. Se observa el principal efecto del ácido cítrico que actúa como acidulante del medio y secuestra los iones metálicos $(\mathrm{Cu}++)$ y del ácido ascórbico que reduce las $o$-quinonas a difenoles incoloros de baja reactividad (Yoruk \& Marshall, 2003).

La muestra AC 0,2\% + TT tuvo mayor influencia en la reducción de la actividad enzimática, a diferencia de las muestras sometidas a los tratamientos TT y AC 1,0 $\%$, evidenciando el efecto combinado de un tratamiento físico y uno químico en el antipardeamiento enzimático que pueden ser aplicados para alargar la vida útil de los productos mínimamente procesados.

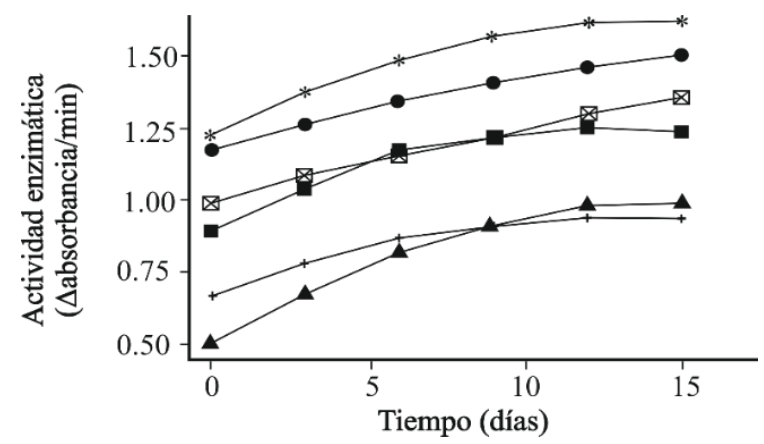

Figura 3. Actividad de la Polifenol Oxidasa en los seis tratamientos evaluados en las muestras de palta almacenada a $4{ }^{\circ} \mathrm{C}$. Los símbolos en la Figura 3 indican, $\bullet$ : TT, $\mathbf{\Delta}:$ AC $0,2 \%+$ TT, $\mathbf{~ : ~ A C ~} 1,0 \%,+$ : AA $0,75 \%, \bigotimes$ : TPS $1,0 \%$ y *: C.

\section{Prueba de preferencia ampliada}

La prueba de preferencia ampliada se llevó a cabo con las muestras AC $0,2 \%+$ TT, AA $0,75 \%$, TPS $1,0 \%$ y AC $1,0 \%$. La prueba de Friedman evidenció que las muestras difieren significativamente $(p<0,05)$ en cuanto a la preferencia, por lo que se efectuó la prueba de Wilcoxon (Tabla 2) donde se observó que no existió diferencia significativa $(\mathrm{p}<0,05)$ entre los tratamientos AC $0,2 \%$ + TT y AA $0,75 \%$, ni entre los tratamientos TPS $1,0 \%$ y AC $1,0 \%$.

Tabla 2. Nivel de significancia de la prueba de comparación múltiple.

\begin{tabular}{lccc}
\hline & AA 1,0 \% & TPS 1,0 \% & AC 0,2 \% + TT \\
\hline TPS 1,0 \% & p-valor $=0,005$ & - & - \\
\hline AC 0,2 \% + TT & n.s & p-valor $=0,0005$ & - \\
\hline AC 1,0 \% & p-valor $=0,001$ & n.s. & p-valor $=0,0009$ \\
\hline
\end{tabular}

n.s: no existe diferencia significativa 
El orden de la preferencia, medida como la suma de las calificaciones de los consumidores, ordenados decrecientemente, fueron las muestras sometidas a los tratamientos AC 0,2\% + TT y AA 0,75\%, AC 1,0\% y TPS $1,0 \%$; siendo el tratamiento AC $0,2 \%+$ TT el más preferido por los consumidores.

\section{CONCLUSIONES}

La luminosidad ( $\mathrm{L}^{*}$ ) de la palta disminuye durante el tiempo de almacenamiento en refrigeración a $4{ }^{\circ} \mathrm{C}$; el valor cromático $\mathrm{a}^{*}$ aumenta y $\mathrm{b}^{*}$ disminuye, producto del pardeamiento enzimático. La aplicación de los tratamientos anti-pardeantes reduce el porcentaje de oscurecimiento y el cambio de color total de las muestras de palta cortadas en trozos y almacenas en refrigeración durante 2 semanas. Debido a la inactivación parcial de la Polifenol Oxidasa (PPO), causante del pardeamiento enzimático. Siendo ordenados de mayor a menor, en cuando a efectividad, de la siguiente manera: tratamiento térmico a $45{ }^{\circ} \mathrm{C}+$ ácido cítrico al 0,2 \% (AC 0,2\% + TT), ácido ascórbico al 0,75\% (AA 0,75\%), tripolifosfato de sodio al 1,0 \% (TPS 1,0\%), ácido cítrico al 1,0\% (AA $1,0 \%)$, tratamiento térmico a $45{ }^{\circ} \mathrm{C}(\mathrm{TT})$ y la muestra control (C), respectivamente. La prueba sensorial con los consumidores evidenció que la muestra AC 0,2\% + TT fue la más preferida por los consumidores en cuanto a sabor.

\section{Conflictos de intereses}

Los autores firmantes del presente trabajo de investigación declaran no tener ningún potencial conflicto de interés personal o económico con otras personas $\mathrm{u}$ organizaciones que puedan influir indebidamente con el presente manuscrito.

\section{Contribuciones de los autores}

Preparación y ejecución: JL, GP; Desarrollo de la metodología: JL, GP; Concepción y diseño: JL, GP; Edición del artículo: JL, GP; Supervisión del estudio: JL.

\section{REFERENCIAS}

- Bustos, M. C., Mazzobre, M. F., \& Buera, M. P.
(2015). Stabilization of refrigerated avocado pulp: Effect of Allium and Brassica extracts on enzymatic browning. LWT - Food Science and Technology, 61(1), 89-97. https://doi.org/10.1016/j.lwt.2014.11.026

- Chang, T. (2009). An updated review of tyrosinase inhibitors. Int. J. Mol. Sci, 10(6), 2440-2475. https://doi.org/10.3390/ijms 10062440

- Denoya, G.I., Ardanaz, M., Sancho, A. M., Benitez, C. E., Gonzáles, C., \& Guidi, S. (2012). Efecto de la aplicación de tratamientos combinados de aditivos sobre la inhibición del pardeamiento enzimático en manzanas cv. Granny Smith mínimamente procesadas. Revista de Investigaciones Agropecuarias, 38(3), 263-267.

- Gacche, R., Aich, A., Dhole, N. A., \& Ghole, V. S. (2006). Reversible inhibition of polyphenol oxidase from apple using L-cysteine. Indian Journal of Chemical Technology, 13, 459-463.

- Gendel, S. M. (2012). Comparison of international food allergen labeling regulations. Regulatory Toxicology and Pharmacology, 63(2), 279-285. https://doi.org/10.1016/j.yrtph.2012.04.007

- Gonzáles-Aguilar, G. A., Ruiz-Cruz, S., Sotovaldez, H., Vázquez-Ortiz, F., Pacheco-Aguilar, R., \& Yi Wang, C. (2005). Biochemical changes of fresh-cut pineapple slices treated with antibrowning agents. International Journal of Food Technology, 40(4), 377-383. https://doi.org/10.1111/j.1365-2621.2004.00940.x

- Hernández, E. H., \& Briceño, L. (2009). Evaluación del pardeamiento enzimático durante el almacenamiento en congelación del puré de palta ( Persea americana Mill ) Var. Hass. Anales Científicos, 70(4), 1-8.

- Lu, S., Luo, Y., Turner, E., \& Feng, H. (2007). Efficacy of sodium chlorite as an inhibitor of enzymatic browning in apple slices. Food Chemistry, 104(2), 824-829. https://doi.org/10.1016/j.foodchem.2006.12.050

- Moskowits, H. R., Beckley, J. H., \& Resurreccion, A. V. (2012). Sensory and consumer research in food product design and development (2nd ed.). John Wiley \& Sons.

- Oms-Oliu, G., Rojas-Graü, M. A., Alandes, L., Varela, P., Soliva-fortuny, R., Hernando, I., Pérez, I., Fiszman, S., \& Martín-belloso, O. (2010). Recent approaches using chemical treatments to 
preserve quality of fresh-cut fruit: A review. Postharvest Biology and Technology, 57(3), 139148.

https://doi.org/10.1016/j.postharvbio.2010.04.001

- Palou, E., López-Malo, G., Barbosa-Cánovas, G., Welti-Chanes, J., \& Swanson, B. G. (1999). Polyphenoloxidase activity and color of blanched and high hydrostatic pressure treated banana puree. Journal of Food Science, 64(1), 42-45. https://doi.org/https://doi.org/10.1111/j.13652621.1999.tb09857.x

- Soliva, R. C., Elez, P., Sebastián, M., \& Martín, O. (2001). Evaluation of browning effect on avocado purée preserved by combined methods. Innovative Food Science \& Emerging Technologies, 1(4), 261-268.

https://doi.org/https://doi.org/10.1016/S14668564(00)00033-3

- Toledo, L. C., \& Aguirre, C. C. (2016). Enzymatic browning in avocado ( Persea americana ) revisited: History, advances and future perspectives. Critical Reviews in Food Science and Nutrition, $\quad$ 57(18), 3860-3872. https://doi.org/10.1080/10408398.2016.1175416

- Tomás-Barberán, F. A., \& Espín, J. C. (2001). Phenolic compounds and related enzymes as determinants of quality in fruits and vegetables.
Journal of the Science of Food and Agriculture, 81(9), 853-876. https://doi.org/10.1002/jsfa.885

- Watada, A. E., Abe, K., \& Yamuchi, N. (1990). Physiological Activities of Partially Processed Fruits and Vegetables. Food Technology, 44(5), 116-122.

- Weemaes, C., Ludikhuyze, L., Broeck, I. Van Den, \& Hendrickx, M. (1999). Kinetic study of antibrowning agents and pressure inactivation of Avocado polyphenoloxidase. Journal of Food Science, 64(5), 823-827. https://doi.org/https://doi.org/10.1111/j.13652621.1999.tb15920.x

- Woolf, A. B., Wibisono, R., Farr, J., Hallett, I., Richter, L., Oey, I., Wohlers, M., Zhou, J., Fletcher, G. C., \& Requejo-Jackman, C. (2013). Effect of high pressure processing on avocado slices. Innovative Food Science and Emerging Technologies, $\quad 18, \quad 65-73$. https://doi.org/10.1016/j.ifset.2013.02.011

- Yoruk, R., \& Marshall, M. R. (2003). Physicochemical properties and funtion of plant polyphenol oxidase: a review. Journal of Food Biochemistry, 27(5), 361-422. https://doi.org/https://doi.org/10.1111/j.17454514.2003.tb00289.x 